\title{
THE IMPACT OF MORPHINE TREATMENT ON BLADDER CANCER CELL PROLIFERATION AND APOPTOSIS: IN VITRO STUDIES
}

\author{
P. Harper, *, O. Hald', B.A. Lwaleed ${ }^{2}$, A. Kyyaly ${ }^{3}$, D. Johnston ${ }^{4}$, A.J. Cooper ${ }^{5}$, B. Birch ${ }^{1}$ \\ ${ }^{1}$ Faculty of Medicine, University of Southampton, Southampton SO17 1BJ, UK \\ ${ }^{2}$ Faculty of Health Sciences, University of Southampton, Southampton SO17 1BJ, UK \\ ${ }^{3}$ Department of Clinical and Experimental Sciences, Faculty of Medicine, \\ University of Southampton, Southampton SO17 1BJ, UK \\ ${ }^{4}$ Biomedical Imaging Unit, University of Southampton, Southampton SO17 1BJ, UK \\ ${ }^{5}$ Faculty of Pharmacy and Biomedical Sciences, University of Portsmouth, Portsmouth PO1 2UP, UK
}

\begin{abstract}
Aim: The aim of this study was to determine the effect of morphine on bladder cancer cell proliferation and apoptosis in vitro. Materials and Methods: MTT assay was used to measure percentage growth of RT-112 human bladder cancer cells after 72 hours of morphine/morphine + naloxone treatment. Expression of $\mu$-opioid receptors was assessed by Western blot and finally, apoptotic assay with CellEvent Caspase-3/7 Green Detection Reagent was carried out using confocal microscopy. Results: The MTT assays showed that morphine increased RT-112 cell growth. Naloxone inhibited this growth enhancing effect. Western blot analysis regarding $\mu$-opioid receptor expression in RT-112 cells remains inconclusive. Morphine was also found to decrease the rate of apoptosis of RT-112 cells, an effect which naloxone inhibited. Conclusions: This study provides evidence that morphine, at clinically relevant doses, causes RT-112 bladder cancer cell proliferation, possibly opioid receptor mediated and at least some of this effect might be due to decreased apoptosis. Clinically, this suggests that in patients with bladder cancer, managing pain with morphine might have detrimental consequences on patient outcomes and alternative pain relief should be considered if possible.
\end{abstract}

Key Words: bladder cancer, morphine, cell proliferation, $\mu$-opioid receptor, apoptosis.

Bladder cancer is the ninth most common cancer worldwide, encompassing several types of cancer which arise from the urothelium. According to GLOBOCAN estimates, around 430,000 new bladder cancer cases and 165,000 bladder cancer deaths occurred globally in 2012 [1]. Morphine plays a role in the management of bladder cancer, specifically, in peri-operative analgesia during tumour resections and radical cystectomy for more advanced disease. However, in recent years, evidence has come to light suggesting that opioids might be undesirable in cancer management.

A number of retrospective clinical studies have demonstrated a lower incidence of cancer recurrence after regional anaesthesia and reduced use of opioids after surgery [2-4]. Laboratory-based studies have reported large amounts of conflicting evidence regarding morphine influence on cell proliferation, apoptosis, invasion/migration and angiogenesis.

The anti-proliferative effects of morphine on cancer cells have been demonstrated in various cell lines including MCF-7 breast cancer cells (due to the activation of opioid receptors) [5], T47D human breast cancer cells (despite lacking opioid receptors) [6], PC9 lung cancer cells (due to the inhibition of NF-kB and TNF-a) [7] and finally in LNCaP, DU145 and PC3 human prostate cancer cells [8]. On the contrary, pro-proliferative effects of morphine have been noted in EL-4 leukaemia cells in mice (although this may have been secondary to immuno-

Submitted: April 27, 2018.

*Correspondence: E-mail: ph2e13@soton.ac.uk Abbreviations used: MTT - methylthiazolyldiphenyl-tetrazolium bromide. suppression) [9], K562 human leukaemia cells [10] and HT-29 colon cancer cells (through the increased secretion of the metastasising protein urokinase-type plasminogen activator) [11].

Similar uncertainty has been shown when looking at morphine effect on cancer cell apoptosis. Anti-apoptotic effects have been demonstrated in SH-SY5Yneuroblastoma cells through a PI3K-signalling cascade [12]. However, using this same cell line, a different study found morphine to induce apoptosis through activation of the mitochondrial death pathway [13]. Pro-apoptotic effects have also been demonstrated in small cell lung cancer cells (due to a reduction in protein kinase $\mathrm{C}$ activity) [14] and Jurkat leukaemia cells [15].

This study aims to determine the in vitro effects of morphine on bladder cancer cell proliferation and apoptosis. It is hypothesised that morphine, at clinically relevant concentrations, will enhance bladder cancer cell proliferation, possibly due to an inhibitory effect on apoptosis through an opioid receptor-mediated mechanism.

\section{MATERIALS AND METHODS}

Reagents. Unless otherwise stated, all reagents were purchased from Sigma-Aldrich ${ }^{\mathrm{TM}}$, UK: Morphine sulphate salt pentahydrate; Naloxone methiodide; methylthiazolyldiphenyl-tetrazolium bromide (MTT); RPMI medium; foetal calf serum; $1 \%$ L-Glutamine-PenicillinStreptomycin solution; $0.4 \%$ trypan blue; phosphate buffered saline; Dimethyl sulfoxide; Sodium dodecyl sulphate. $\mu$-opioid primary antibody (ab10275) and secondary antibody (ab205718) were from Abcam, UK; CellEvent Caspase-3/7 Green Detection Reagent was from Thermofisher Scientific ${ }^{\mathrm{TM}}$, UK. 
Cell culture. RT-112 human urinary bladder transitional carcinoma cells were maintained in RPMI medium supplemented with $10 \%$ foetal calf serum and $1 \%$ L-Glutamine-Penicillin-Streptomycin solution. The cells were incubated at $37^{\circ} \mathrm{C}$ in a humidified atmosphere with $5 \% \mathrm{CO}_{2}$ and passaged when they reached $90 \%$ confluence.

MTT assay. RT-112 cells $\left(1 \cdot 10^{5}\right)$ were seeded into the 60 inner wells of 96 -well microplates containing $100 \mu \mathrm{l}$ of culture medium. Phosphate buffered saline was added to the outer 36 wells. Plates were incubated at $37^{\circ} \mathrm{C}$ in $5 \% \mathrm{CO}_{2}$ for 24 hours. Doubling dilutions of treatment/blocker was then added to the wells $(n=6)$ except for the eleventh column which was kept as a control. Plates were incubated for a further 72 hours. $50 \mu$ l of MTT solution was added to each well. Precipitated formazan crystals were dissolved by adding $100 \mu \mathrm{l}$ dimethyl sulfoxide. The plates were inserted into the spectrophotometer and read at $570 \mathrm{~nm}$ and $630 \mathrm{~nm}$. Each MTT assay was repeated 4 times.

Immunoblotting. RT-112 cells were lysed in breaking buffer ( $50 \mathrm{mM}$ sodium phosphate, $1 \mathrm{mM}$ PMSF, $1 \mathrm{mM}$ EDTA, $5 \%$ glycerol). $10 \mu \mathrm{g}$ aliquots of protein sample were mixed with $6 \times$ SDS loading buffer, incubated for 10 minutes at $75^{\circ} \mathrm{C}$ and loaded on a 4-20\% gradient polyacrylamide gel along with the molecular weight marker. The proteins were then transferred onto a nitrocellulose membrane at $30 \mathrm{~mA}$ for 2 hours. Following SDS-polyacrylamide gel electrophoresis and electroblotting, membranes were blocked using a $5 \%$ milk solution for 2 hours. Following this, they were incubated with the $\mu$-opioid primary antibody $(1: 1000)$ and subsequently the secondary antibody for 2 hours each (1:1000). Antibody-labelled protein bands were visualised by enhanced chemiluminescent detection. Coomassie staining was used to exhibit the protein profile of the RT- 112 cells.

Apoptosis assay. RT-112 cells $\left(1 \cdot 10^{5}\right)$ were seeded into a $\mu$-Slide 8 -Well Glass Bottom dish. Cells were incubated at $37^{\circ} \mathrm{C}$ in $5 \% \mathrm{CO}_{2}$ for 24 hours and then treated with morphine/naloxone. The dishes were incubated for a further 72 hours. After removing the media from the cells, CellEvent Caspase-3/7 Green Detection Reagent (Thermofisher Scientific ${ }^{\mathrm{TM}}$, UK) was added to the cells. Cells were imaged using confocal microscopy.

Statistical analysis. MTT assay - results from the plate reader were analysed using Graphpad Prism (Graphpad Software, San Diego, CA). $p$ values were calculated by comparing the control average with an experimental average using Student's paired $t$-tests. The $62.5 \mu \mathrm{M}$ experimental value was deemed the most clinically relevant and therefore was appropriate for statistical analysis to compare against the control $[16,17]$.

Apoptotic assay - the number of apoptotic cells showing bright green nuclei were calculated as a percentage of the total number of cells and analysed using Graphpad Prism.

\section{RESULTS}

Growth response of $R T-112$ cells to exogenous morphine. Morphine doubling dilutions $(250 \mu \mathrm{M} \rightarrow 0.977 \mu \mathrm{M})$ were performed across a 96well plate. As shown in Fig. 1, the percentage growth of the treated cells was greater than that of the control cells. This effect diminished as morphine concentrations decreased. At the $62.5 \mu \mathrm{M}$ (clinically relevant) concentration, there was $\sim 7 \%$ increase in cell growth $(p=0.0079)$, whereas at the lowest concentrations of morphine, there was negligible effect.

Growth response of $R T-112$ cells to morphine after pre-treatment with clinically relevant naloxone dose. A $50 \mu \mathrm{M}$ blanket dose of naloxone was applied across a 96 -well plate, 15 minutes prior to morphine doubling dilutions $(250 \mu \mathrm{M} \rightarrow 0.977 \mu \mathrm{M})$. Initially, at the higher doses of morphine, percentage growth was higher than that of the control (Fig. 2). At the $62.5 \mu \mathrm{M}$ morphine dose, the increase in growth was $\sim 2 \%$ which was not significantly different from the control ( $p=0.7445$ ) and this was most likely due to the competitive binding of naloxone at the opioid receptors. As morphine concentrations dropped below the $50 \mu \mathrm{M}$ naloxone dose, percentage growth decreased to below that of the control.

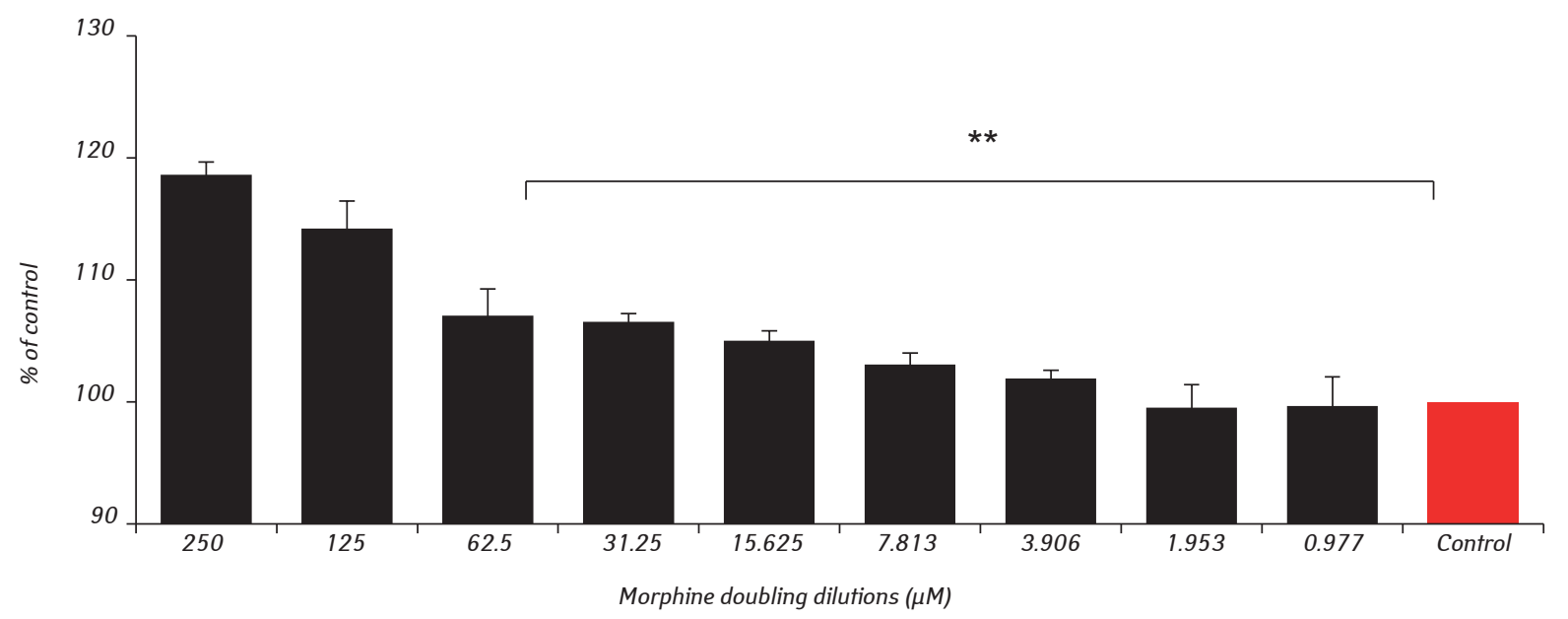

Fig. 1. Growth response of RT-112 cells to exogenous morphine. Bar chart showing the percentage difference between growth of the treated cells and control cells Statistics is calculated for $62.5 \mu \mathrm{M}$ compared to control using Student's paired $t$-test. ${ }^{\star \star} p<0.05$ 


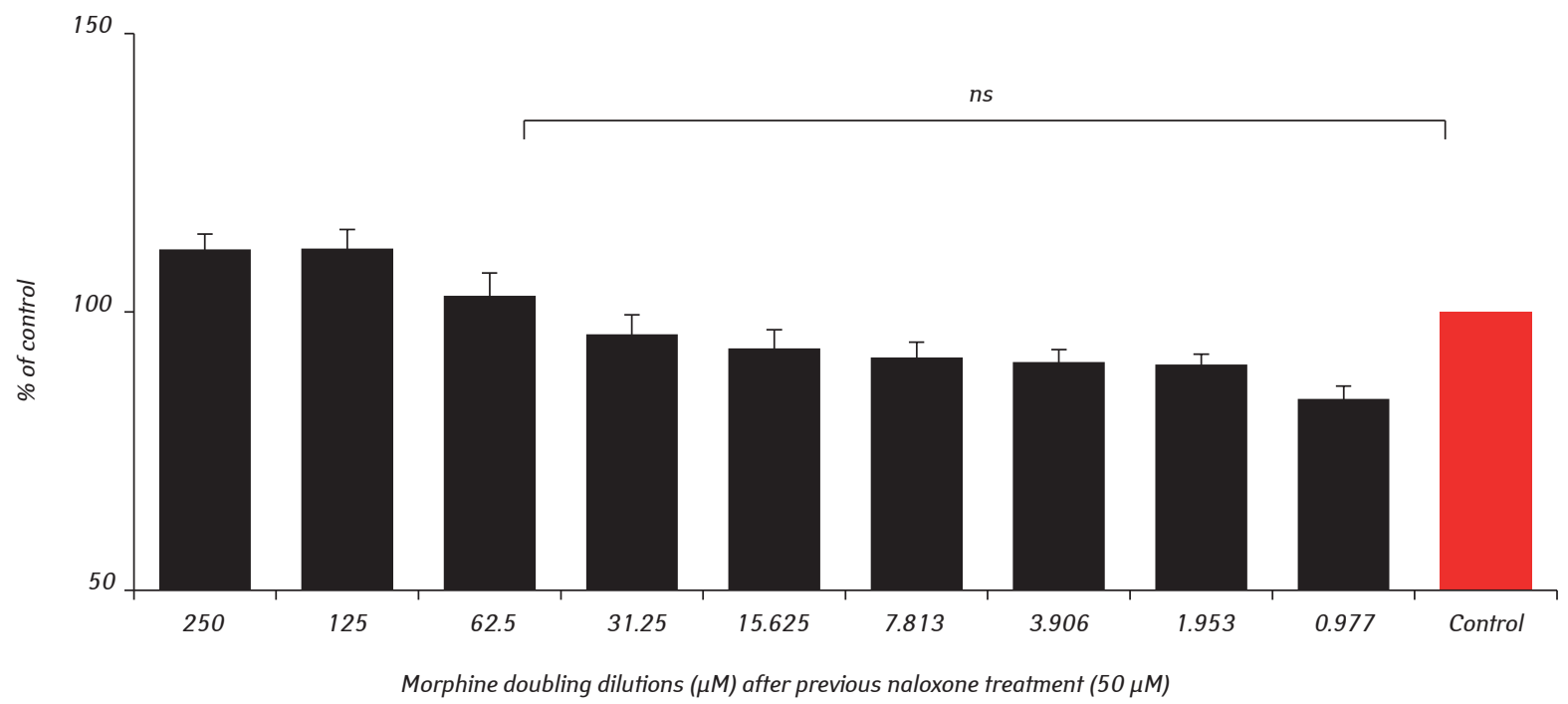

Fig. 2. Growth response of RT-112 cells to morphine after pre-treatment with clinically relevant naloxone dose $50 \mu \mathrm{M}$. Bar chart showing the percentage difference between growth of the treated cells and growth of the control cells Statistics is calculated for $62.5 \mu \mathrm{M}$ compared to control using Student's paired $t$-test. $n s-$ non-significant $(p>0.05)$

$R T$-112 cell expression of the $\mu$-opioid receptor. The next step in this study was to determine if the $\mu$-opioid receptor was expressed by RT-112 cells. In dot blot experiments with $\mu$-opioid receptor antibody only a weak signal was obtained. While in Western blot analysis a weak signal corresponded to a molecular weight of 25-30 kDa while the predicted molecular weight of the $\mu$-opioid receptor is $45 \mathrm{kDa}$ (data not shown).

Effect of morphine on RT-112 cell apoptosis. Confocal imaging was used to determine whether morphine, with and without naloxone, had any effect on RT-112 cell apoptosis. Fig. 3, a displays the most representative image of each well in the 8-well glass dish and the treatment that each well received. Note that many images were taken across each well, all of which were analysed during cell counting. Fig. $3, b$ quantifies the findings from these images. The percentage of apoptotic cells was $\sim 13 \%$ in the morphine $(50 \mu \mathrm{M})$ group $([p=0.1345), \sim 28 \%$ in the morphine $(50 \mu \mathrm{M})+$ naloxone $(100 \mu \mathrm{M})$ group $(p=0.6594)$ and $\sim 24 \%$ in the control group. Despite not achieving statistical significance, these results remain interesting and informative and perhaps shed some light on how morphine exerts its growth-inducing effects.

\section{DISCUSSION}

This study adds further evidence to the debate that morphine may be undesirable in the management of pain, in patients with bladder cancer (transitional cell carcinoma). Fig. 1 shows that clinically relevant doses of morphine cause RT-112 cell proliferation, corresponding to $5-10 \%$ growth increase. Other studies have found comparable results, for example, with human glioma T98G cells [18] and human colon cancer HCT116 cells (believed to be caused by modulation of cell proliferation and/or apoptosis) [19]. In these studies the concentrations of morphine used were below $1 \mu \mathrm{M}$, however, evidence suggests that in peri-operative patients receiving morphine, plasma

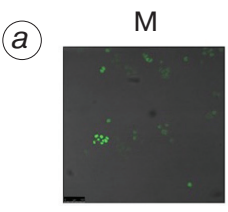

C

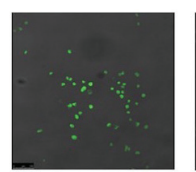

(b)

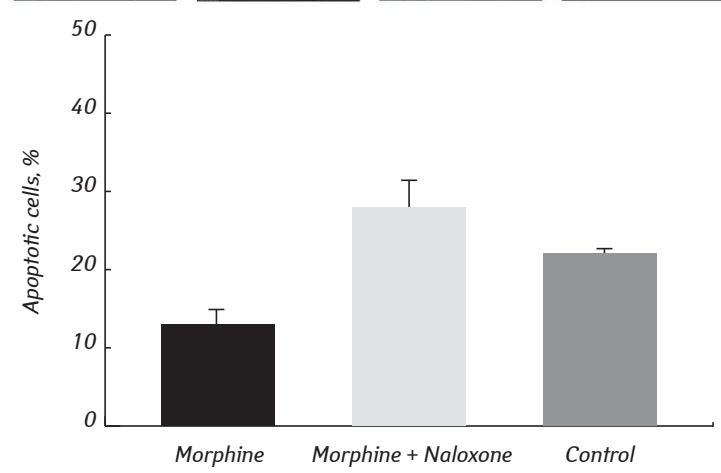

Fig. 3. The effect of morphine on RT-112 cell apoptosis. a - Confocal images showing the number of apoptotic cells in each well (fluorescent green). $\mathrm{M}-$ morphine-treated well; $\mathrm{M}+\mathrm{N}-$ morphine- and naloxone-treated well; $\mathrm{C}-\mathrm{control}$ well. $b-$ Bar chart showing the percentage of apoptotic cells in the different treatment groups

concentrations can reach higher than $70 \mu \mathrm{M}$ [16]. This is why $62.5 \mu \mathrm{M}$ was deemed a clinically relevant concentration in these experiments.

When morphine and naloxone were co-administered, they demonstrated a competitive relationship. Fig. 2 shows that when morphine concentration exceeded naloxone concentration, the result was RT-112 cell proliferation. However, when naloxone concentration exceeded morphine concentration, cell growth was reduced. Percentage growth ranged from $+10 \%$ to $-15 \%$ across the plate. This suggests that the growth-enhancing effects of morphine are opioid receptor-mediated. 
Involvement of the $\mu$-opioid receptor in regulating cancer progression has been reported in several laboratory studies [20-22]. To determine whether RT-112 cells express $\mu$-opioid receptors, several immunoblotting experiments were performed. Nevertheless, Western blot analysis regarding $\mu$-opioid receptor expression in RT-112 cells in our study remained inconclusive. Finally, in our experiments morphine reduces the rate of apoptosis in RT-112 cells, which may partly explain how morphine causes RT-112 cell proliferation. Naloxone inhibited morphine anti-apoptotic effect. Lin et al. reported similar findings where morphine inhibited doxorubicin-induced neuroblastoma cell apoptosis by the inhibition of reactive oxygen species generation, mitochondrial cytochrome $\mathrm{c}$ release and NF-kB transcriptional activation [23]. Morphine has also been shown to protect lymphocytic cells from apoptotic lysis, mediated by opioid receptors [24].

In our study, morphine, at clinically relevant doses, caused RT-112 bladder cancer cell proliferation. The results support an opioid receptor mediated effect and at least some of this might be due to decreased apoptosis. This has not been reported previously for a bladder cancer cell line but concurs with other studies carried out on different cancer cell lines. Clinically, the use of morphine to manage pain in patients with bladder cancer may need to be carefully considered.

\section{FUNDING}

The study was funded by the University of Southampton.

\section{ACKNOWLEDGEMENTS}

The authors thank members of staff in the Departments of Urology and Clinical and Experimental Sciences at University Hospital Southampton NHS Foundation Trust for their support.

\section{REFERENCES}

1. Antoni S, Ferlay J, Soerjomataram I, et al. Bladder cancer incidence and mortality: a global overview and recent trends. Eur Urol 2017; 71: 96-108.

2. Biki B, Mascha E, Moriarty DC, et al. Anesthetic technique for radical prostatectomy surgery affects cancer recurrence: a retrospective analysis. Anesthesiology 2008; 109: 180-7.

3. Exadaktylos AK, Buggy DJ, Moriarty DC, et al. Can anesthetic technique for primary breast cancer surgery affect recurrence or metastasis? Anesthesiology 2006; 105: 660-4.

4. Lin L, Liu C, Tan H, et al. Anaesthetic technique may affect prognosis for ovarian serous adenocarcinoma: a retrospective analysis. Br J Anaesth 2011; 106: 814-22.

5. Maneckjee R, Biswas R, Vonderhaar BK. Binding of opioids to human MCF-7 breast cancer cells and their effects on growth. Cancer Res 1990; 50: 2234-8.

6. Hatzoglou A, Ouafik L, Bakogeorgou E, et al. Morphine cross-reacts with somatostatin receptor SSTR2 in the T47D human breast cancer cell line and decreases cell growth. Cancer Res 1995; 55: 5632-6.

7. Sueoka E, Sueoka N, Kai Y, et al. Anticancer activity of morphine and its synthetic derivative, KT-90, mediated Copyright @ Experimental Oncology, 2018 through apoptosis and inhibition of NF-kappaB activation. Biochem Biophys Res Commun 1998; 252: 566-70.

8. Kampa M, Bakogeorgou E, Hatzoglou A, et al. Opioid alkaloids and casomorphin peptides decrease the proliferation of prostatic cancer cell lines [LNCaP, PC3 and DU145] through a partial interaction with opioid receptors. Eur J Pharmacol 1997; 335: 255-65.

9. Ishikawa M, Tanno K, Kamo A, et al. Enhancement of tumor growth by morphine and its possible mechanism in mice. Biol Pharm Bull 1993; 16: 762-6.

10. Sergeeva MG, Grishina ZV, Varfolomeyev SD. Morphine effect on proliferation of normal and tumor cells of immune origin. Immunol Lett 1993; 36: 215-8.

11. Nylund $G$, Pettersson A, Bengtsson C, et al. Functional expression of mu-opioid receptors in the human colon cancer cell line, HT-29, and their localization in human colon. Dig Dis Sci 2008; 53: 461-6.

12. Iglesias M, Segura MF, Comella JX, et al. Mu-opioid receptor activation prevents apoptosis following serum withdrawal in differentiated SH-SY5Y cells and cortical neurons via phosphatidylinositol 3-kinase. Neuropharmacology 2003; 44: 482-92.

13. Lin X, Wang YJ, Li Q, et al. Chronic high-dose morphine treatment promotes $\mathrm{SH}-\mathrm{SY} 5 \mathrm{Y}$ cell apoptosis via c-Jun $\mathrm{N}$-terminal kinase-mediated activation of mitochondriadependent pathway. Febs J 2009; 276: 2022-36.

14. Maneckjee R, Minna JD. Opioids induce while nicotine suppresses apoptosis in human lung cancer cells. Cell Growth Differ 1994; 5: 1033-40.

15. Yin D, Woodruff $M$, Zhang $Y$, et al. Morphine promotes Jurkat cell apoptosis through pro-apoptotic FADD/ P53 and anti-apoptotic PI3K/Akt/NF-kappaB pathways. J Neuroimmunol 2006; 174: 101-7.

16. Couper FJ, Logan BK. Drugs and human performance fact sheets. NHTSA, 2004. 100 p.

17. Martin del Campo AF, Granados-Soto V, AguirreBañuelos P, et al. The use of the plasma concentration-effect relationship as a tool for the study of the mechanism of action of naloxone effects on mood and endocrine function. J Psychopharmacol 1997; 11: 361-5.

18. Lazarczyk M, Matyja E, Lipkowski AW. A comparative study of morphine stimulation and biphalin inhibition of human glioblastoma T98G cell proliferation in vitro. Peptides 2010; 31: 1606-12.

19. Nomura Y, Kawaraguchi Y, Sugimoto H, et al. Effects of morphine and fentanyl on 5-fluorouracil sensitivity in human colon cancer HCT116 cells. J Anesth 2014; 28: 298-301.

20. Singleton PA, Moss J. Effect of perioperative opioids on cancer recurrence: a hypothesis. Future Oncol 2010; 6: $1237-42$.

21. Wang CZ, Li XL, Sun S, et al. Methylnaltrexone, a peripherally acting opioid receptor antagonist, enhances tumoricidal effects of 5-Fu on human carcinoma cells. Anticancer Res 2009; 29: 2927-32.

22. Mathew B, Lennon FE, Siegler J, et al. The novel role of the mu opioid receptor in lung cancer progression: a laboratory investigation. Anesth Analg 2011; 112: 558-67.

23. Lin X, Li Q, Wang YJ, et al. Morphine inhibits doxorubicin-induced reactive oxygen species generation and nuclear factor kappaB transcriptional activation in neuroblastoma SH-SY5Y cells. Biochem J 2007; 406: 215-21.

24. Suzuki S, Chuang LF, Doi RH, et al. Morphine suppresses lymphocyte apoptosis by blocking p53-mediated death signaling. Biochem Biophys Res Commun 2003; 308: $802-8$. 